VOL. $21(1980), 189-205$.

\title{
SPACES WITH HOMOGENEOUS NORMS
}

\author{
A.J. PRYDE
}

\begin{abstract}
Spaces with homogeneous norms are closely related to the Beppo Levi spaces of Deny and Lions, to spaces of Riesz potentials, and to Sobolev spaces. In this paper we survey the literature on them, give a broad extension of their definition, and present their basic theory. Many of the properties of Sobolev spaces have their analogues. In fact, the two families are locally equivalent. Spaces with homogeneous norms are especially suited to the study of boundary value problems on $R_{+}^{n}$ for homogeneous elliptic operators with constant coefficients. We will use them extensively in a forthcoming paper to study elliptic partial differential equations with mixed boundary conditions on a smoothly bounded domain.
\end{abstract}

\section{Introduction}

The most common class of function spaces in which to study boundary value problems for elliptic partial differential equations is the class of Sobolev spaces. However, a number of authors have also used spaces related to the Beppo Levi spaces of Deny and Lions [4].

Following initial work by Beppo Levi, Nikodym, Deny and others (see the references in [4]), Deny and Lions defined Beppo Levi spaces as follows. Let $\Omega$ be a connected open subset of $R^{n}$ and $E$ a complete Hausdorff locally convex space continuously embedded in $D^{\prime}(\Omega)$, the space

Received 3 September 1979. Part of this work was included in a $\mathrm{PhD}$ thesis at Macquarie University under the supervision and much appreciated guidance of Alan McIntosh. 
of Schwartz distributions on $\Omega$. For a non-negative integer $m, B L_{m}(E)$ is the space of distributions $u$ satisfying $D^{\alpha} u \in E$ for all partial derivatives $D^{\alpha} u$ of $u$ of order $m$, and is given the coarsest topology for which all the maps $u \rightarrow D^{\alpha} u$ are continuous from $B L_{m}(E)$ into $E$. The closure of $\{0\}$ in $B L_{m}(E)$ is $P^{m-1}(\Omega)$ the space of polynomials on $\Omega$ of degree less than $m$. One of the main results ([4], Theorem 2.1) is that the spaces $B L_{\dot{m}}(E)=B L_{m}(E) / P^{m-1}(\Omega)$ are complete.

It follows that the spaces $B L_{m}(\Omega)=B L_{m}\left(L^{2}(\Omega)\right)$ are Hilbert spaces with norm $\|u\|_{m}$ given by the Dirichlet integral.

$$
\|u\|_{m}^{2}=\sum_{\mid \alpha T=m} \int_{\Omega}\left|D^{\alpha} u\right|^{2} d x .
$$

Deny and Lions used these spaces, with $m=1$, to consider the Dirichlet and Neumann boundary value problems for the Laplacian.

Also with a view to studying the Dirichlet problem for the Laplacian, Hormander and Lions [6] considered the completion $\hat{D}^{m}(\Omega)$ of the space $C_{0}^{\infty}(\Omega)$ with the respect to the norm $(1.1)$. So $\hat{D}^{m}(\Omega)$ can be identified with a closed subspace of $B L_{m}(\Omega)$.

Malgrange [8] improved the results of Hormander and Lions by replacing the Dirichlet integral with a generalised Dirichlet integral corresponding to a Hermitian form of the type $\sum a_{\alpha \beta} \zeta^{\alpha} \bar{\zeta}^{\beta}$ where $|\alpha|=|\beta|=m$ and $\zeta \in c^{n}$

Following on work of Frostman, Riesz, Cartan and Deny (see the references in [2]), Aronszajn and Smith [2] considered, for $0<s<n / 2$, the space $R P^{s}\left(R^{n}\right)$, say, of.Riesz potentials $u=K_{s} f_{f}$ of order $s$ of functions $f \in L^{2}\left(R^{n}\right)$, where

$$
K_{s}(x)=\left(\Gamma(s / 2) \pi^{n / 2} 2^{s}\right)^{-1} \Gamma\left(\frac{n-s}{2}\right)|x|^{s-n} .
$$


The space is provided with the norm $\|u\|_{s}=\|f\|_{L^{2}} \cdot$ Defining the Fourier transform $\hat{f}$ of $f \in L^{1}\left(R^{n}\right)$ by

$$
\hat{f}(\xi)=(2 \pi)^{-n / 2} \int e^{-i x \cdot \xi} f(x) d x
$$

and extending the definition in the usual way to arbitrary $f$ in the Schwartz space $S^{\prime}\left(R^{n}\right)$, it follows that

$$
\hat{K}_{s}(\xi)=|\xi|^{-s} \text {, for } 0<s<n / 2 \text {. }
$$

Hence the norm of $R P^{s}\left(R^{n}\right)$ is also given by

$$
\|u\|_{s}=\left\||\xi|^{s \hat{u}}\right\|_{L^{2}}
$$

Further, since $\left\{|\xi|^{s} \hat{u}: u \in C_{0}^{\infty}\left(R^{n}\right)\right\}$ is dense in $L^{2}\left(R^{n}\right)$ (Lemma 2.I below), it follows that $R P^{s}\left(R^{n}\right)$ is a completion of $C_{0}^{\infty}\left(R^{n}\right)$. Also, as is proved instein([20], v.l.I and v.1.2), for example, if $0<s<n / 2$ and $1 / q=1 / 2-s / n$, then

$$
R P^{s}\left(R^{n}\right) \subset L^{q}\left(R^{n}\right)
$$

and the embedding is continuous.

Aronszajn and Smith [3] showed that for all $s \geq 0$ an equivalent norm to $(1.5)$ is given by the generalized Dirichlet integral

$$
\begin{aligned}
& \|u\|_{s}^{2}=\sum_{\mid \alpha \uparrow=s} \int\left|D^{\alpha} u\right|^{2} d x \text {, if } s \text { is an integer, } \\
& \|u\|_{s}^{2}=\sum_{|\alpha|=[s]} \iint \frac{\left|D^{\alpha} u(x)-D^{\alpha} u(y)\right|^{2}}{|x-y|^{n+2 s-2[s]}} d x d y \text {, otherwise, }
\end{aligned}
$$

where [s] is the largest integer less than $s$. However, ([3], p. 404), if $s \geq n / 2, C_{0}^{\infty}\left(R^{n}\right)$ normed by (1.5) or (1.7) is not a functional space, since norm convergence does not imply pointwise convergence of a subsequence in any reasonable sense. For this reason, Aronszajn and Smith turned their attention to Bessel potentials.

The Sobolev space $H^{s}\left(R^{n}\right)$, for $s>0$, is the space of Bessel 
potentials $u=G_{s} * f$ of order $s$ of functions $f \in L^{2}\left(R^{n}\right)$, where

$$
\hat{G}_{s}(\xi)=\left(1+|\xi|^{2}\right)^{-s / 2} .
$$

This defines a functional completion of $C_{0}^{\infty}\left(R^{n}\right)$ with the norm

$$
\|u\|_{s}=\left\|\left(I+|\xi|^{2}\right)^{s / 2} \hat{u}\right\|_{L^{2}\left(R^{n}\right)} .
$$

Moreover, the homogeneous norm (1.5) and the Sobolev norm (1.9) are equivalent on $C_{0}^{\infty}(\Omega)$ for any bounded open subset $\Omega$ of $R^{n}$ ([3], p. 408).

For arbitrary real $s, H^{s}\left(R^{n}\right)$ is the space of distributions $u \in S^{\prime}\left(R^{n}\right)$ for which $\hat{u} \in L_{1 \circ c}^{1}\left(R^{n}\right)$ and $\left(1+|\xi|^{2}\right)^{s / 2} \hat{u} \in L^{2}\left(R^{n}\right)$. It is a completion of $C_{0}^{\infty}\left(R^{n}\right)$ with the norm (1.9). For an arbitrary open subset $\Omega$ of $R^{n}, \stackrel{\circ}{H^{s}}(\Omega)$ denotes the subspace of $H^{s}\left(R^{n}\right)$ of distributions with support in $\bar{\Omega}$, and $H^{S}(\Omega)$ the space of restrictions to $\Omega$ of distributions in $H^{s}\left(R^{n}\right)$ with the infimum norm.

Shamir [17] and [18] defined the space $W^{S}(\Omega)$, for an open subset $\Omega$ of $R^{n}$ and $s \geq 0$, to be the completion of $C_{0}^{\infty}(\bar{\Omega})$ with respect to the norm (1.7). His purpose was to study elliptic partial differential equations with mixed boundary conditions.

It is the purpose of this paper to extend the definition of these spaces based on Riesz potentials to the case of arbitrary real $s$ when $\Omega=R^{n}, R_{+}^{n}$ or $R_{-}^{n}$, and to present some of their important properties. By $R_{+}^{n}\left(R_{-}^{n}\right)$ is meant the subset of $R^{n}$ determined by $x_{n}>0 \quad\left(x_{n}<0\right)$ where $x=\left(x_{1}, \ldots, x_{n}\right)=\left(x^{\prime}, x_{n}\right) \in R^{n}$. The notation we use for the spaces is $Z^{S}(\Omega)$ and we refer to them as spaces with homogeneous norms.

When handling elliptic equations with mixed boundary conditions, various authors including Peetre [11], [12], Shamir [17] and [19], and 
Vishnik and Eskin [21] and [22], have made use of known a priori estimates for the ordinary boundary conditions, and reduced similar estimates for the mixed boundary conditions to estimates for Wiener Hopf operators. As indicated in Pryde [15] and as we plan to demonstrate in a future paper, this reduction can be generalized and greatly simplified using spaces with homogeneous norms. They appear to be the natural spaces in which to consider boundary value problems in $R_{+}^{n}$ for homogeneous elliptic operators with constant coefficients.

To indicate the use of these spaces we state a theorem to be proved in a later paper. For this we consider an operator $A(D)$ and boundary system $\left(B_{j}(D)\right)$ as follows. With $\xi=\left(\xi_{1}, \ldots, \xi_{n}\right)=\left(\xi^{\prime}, \xi_{n}\right) \in R^{n}$, let

$$
A(\xi)=\sum_{k=0}^{m} a_{k}\left(\xi^{\prime}\right) \xi_{n}^{k} \text { and } B_{j}(\xi)=\sum_{k=0}^{m} b_{j k}\left(\xi^{\prime}\right) \xi_{n}^{k}, \quad 0 \leq j \leq p-1 \text {, }
$$

where $a_{k}\left(\xi^{\prime}\right)$ and $b_{j k}\left(\xi^{\prime}\right)$ are positively homogeneous of order $m-k$ and $m_{j}-k$ respectively, and continuous for $\xi^{\prime} \neq 0$. For each $\xi^{\prime} \neq 0$, let

$$
A^{+}\left(\xi^{\prime}, z\right)=\prod_{k=1}^{r\left(\xi^{\prime}\right)}\left(z-z_{k}\left(\xi^{\prime}\right)\right)
$$

where the $z_{k}\left(\xi^{\prime}\right)$ are the roots of $A\left(\xi^{\prime}, z\right)$ with positive imaginary part. Let $r=\max r\left(\xi^{\prime}\right)$ and define the $p \times r$ matrix $B\left(\xi^{\prime}\right)=\left(B_{j k}\left(\xi^{\prime}\right)\right)$ by

$$
B_{j}\left(\xi^{\prime}, z\right) \equiv \sum_{k=0}^{r-1} B_{j k}\left(\xi^{\prime}\right) z^{k}\left(\bmod A^{+}\left(\xi^{\prime}, z\right)\right), \quad 0 \leq j \leq p-1,
$$

where $B_{j k}\left(\xi^{\prime}\right)=0$ for $r\left(\xi^{\prime}\right) \leq k \leq r-1$.

THEOREM 1.12. Suppose $A$ is elliptic and let $B$ denote the operator

$$
\left(B_{j}(D)\right): z_{\operatorname{ker} A}^{s}\left(R_{+}^{n}\right) \rightarrow \prod_{j=0}^{p-1} z^{s-m_{j}-\frac{z}{2}}\left(R^{n-1}\right)
$$

where the subscript $\operatorname{ker} A$ denotes the kermel of $A$ in the indicated 
space. Then $B$ is left (might) invertible if and only if rank $B\left(\xi^{\prime}\right)=r\left(\xi^{\prime}\right)$ (respectively $p$ ) for all $\xi^{\prime} \neq 0$.

\section{Definitions}

For real $s$ let $P^{S}\left(R^{n}\right)$ denote the subspace of $S^{\prime}\left(R^{n}\right)$ consisting of polynomials of degree less than or equal to $s$ and set

$$
D^{s}\left(R^{n}\right)=\left\{u \in C_{0}^{\infty}\left(R^{n}\right): \int u(x) p(x) d x=0 \text { for all } p \in P^{-\varepsilon-n / 2}\left(R^{n}\right)\right\}
$$

with the topology of $S\left(R^{n}\right)$.

LEMMA 2.1. For each real $s$, the operator $|\nabla|^{s}: D^{s}\left(R^{n}\right) \rightarrow L^{2}\left(R^{n}\right)$, defined by $\left(|\nabla|^{s} u\right)^{\wedge}(\xi)=|\xi|^{s} \hat{u}(\xi)$, is bounded and has dense range.

Proof. The boundedness is clear for $s \geq 0$. If $s<0$, set $1-s-n / 2=r+\delta$ where $x$ is an integer and $0 \leq \delta<1$. Then $u \in D^{s}\left(R^{n}\right)$ implies $p(D) \hat{u}=0$ for all $p \in P^{r-1}\left(R^{n}\right)$, and by Schwartz's lemma ([5], Theorem I.A.8), $|\xi|^{-2} \hat{u}$ is bounded on the unit ball by $M=\sup _{|\xi| \leq 1}|\hat{u}(\xi)| \leq\|u\|_{L^{1}}$.

Hence,

$$
\begin{aligned}
\left\||\nabla|^{s} u\right\|_{L^{2}}^{2} & \leq \int_{|\xi| \leq 1}|\xi|^{2 s}|\hat{u}(\xi)|^{2} d \xi+\int_{|\xi|>1}|\hat{u}(\xi)|^{2} d \xi \\
& \leq M^{2} \int_{|\xi| \leq 1}|\xi|^{2-n-2 \delta} d \xi+\|u\|_{L^{2}}^{2} \\
& \leq c\|u\|_{L^{1}}^{2}+\|u\|_{L^{2}}^{2} .
\end{aligned}
$$

Since the topology of $S\left(R^{n}\right)$ is finer than that of $L^{\perp}\left(R^{n}\right)$ and $L^{2}\left(R^{n}\right)$, boundedness is proved.

To show the range is dense, let $m$ be a positive integer such that $t=2 m+s \geq 0$. Then $|\nabla|^{t}\left(1+|\nabla|^{2}\right)^{-t / 2}: L^{2}\left(R^{n}\right) \rightarrow L^{2}\left(R^{n}\right)$ is bounded, selfadjoint and injective, and therefore has dense range. But $C_{0}^{\infty}\left(R^{n}\right)$ is dense in $H^{t}\left(R^{n}\right)=\left(1+|\nabla|^{2}\right)^{-t / 2} L^{2}\left(R^{n}\right)$, giving $|\nabla|^{t} C_{0}^{\infty}\left(R^{n}\right)$ is dense in 
$L^{2}\left(R^{n}\right)$. Since $|\nabla|^{2 m} C_{0}^{\infty}\left(R^{n}\right) \subset D^{s}\left(R^{n}\right),|\nabla|^{s} D^{s}\left(R^{n}\right)$ is dense in $L^{2}\left(R^{n}\right)$

Now the (sesquilinear) pairing between $S\left(R^{n}\right)$ and $S^{\prime}\left(R^{n}\right)$, given by the usual extension of the $L^{2}$ inner product, induces a pairing between $D^{s}\left(R^{n}\right)$ and $S^{\prime}\left(R^{n}\right) / P^{-s-n / 2}\left(R^{n}\right)$. For, since $P^{-s-n / 2}\left(R^{n}\right)$ is finite dimensional, it is the annihilator of $D^{s}\left(R^{n}\right)$. From the lemma, by taking duals, we obtain a bounded injection

$$
|\nabla|^{s}: L^{2}\left(R^{n}\right)+S^{\prime}\left(R^{n}\right) / P^{-s-n / 2}\left(R^{n}\right)
$$

DEFINITION 2.3. Take $s$ real and $\Omega=R_{+}^{n}$ or $R_{-}^{n}$. Then $2^{s}\left(R^{n}\right)$ denotes $|\nabla|^{-s} L^{2}\left(R^{n}\right)$ with the homogeneous norm (1.5), a Hilbert space continuously embedded in $S^{\prime}\left(R^{n}\right) / P^{s-n / 2}\left(R^{n}\right) ; Z^{S}(\Omega)$ denotes the subspace of $Z^{s}\left(R^{n}\right)$ consisting of factor classes containing a distribution with support in $\bar{\Omega}$; and $Z^{S}(\Omega)$ denotes the space of restrictions to $\Omega$ of members of $Z^{s}\left(R^{n}\right)$, with the infimum norm.

\section{Basic properties}

By considering the sequence

$$
D^{s}\left(R^{n}\right) \stackrel{|\nabla|^{s}}{\longrightarrow} L^{2}\left(R^{n}\right) \stackrel{|\nabla|^{-s}}{\longrightarrow} S^{\prime}\left(R^{n}\right) / P^{s-n / 2}\left(R^{n}\right)
$$

we see that $D^{s}\left(R^{n}\right)$ can be identified with a dense subspace of $Z^{\mathcal{S}}\left(R^{n}\right)$. Similarly, for $s \geq 0$ there is a continuous dense embedding $H^{s}\left(R^{n}\right) \subset Z^{s}\left(R^{n}\right)$. For arbitrary $s$, the isomorphisms $|\nabla|^{s}: L^{2}\left(R^{n}\right) \rightarrow Z^{-s}\left(R^{n}\right)$ with inverse $|\nabla|^{-s}$, say, induce a pairing

$$
\langle u, v\rangle=\left(|\nabla|^{s} u,|\nabla|^{-s} v\right)_{L^{2}\left(R^{n}\right)} \text { on } z^{s}\left(R^{n}\right) \times z^{-s}\left(R^{n}\right),
$$

which is an extension of the $L^{2}$ inner product on the dense subspace $D^{s}\left(R^{n}\right) \times D^{-s}\left(R^{n}\right)$. Hence, for $s<0$, we have a continuous dense 
embedding $Z^{s}\left(R^{n}\right) \subset H^{s}\left(R^{n}\right)$.

Let $R_{\Omega}: z^{s}\left(R^{n}\right) \rightarrow z^{s}(\Omega)$ denote the (bounded) restriction operator. If $\Omega^{\prime}=R^{n}-\bar{\Omega}$, the kernel of $R_{\Omega^{\prime}}$ is $\stackrel{\circ}{Z}^{s}(\Omega)$, which is therefore a Hilbert space. We show that $Z^{\mathcal{S}}(\Omega)$ is also a Hilbert space by constructing a bounded right inverse $P_{\Omega}$ of $R_{\Omega}$.

For this, take a positive integer $m \geq 2+|s|$ and let $\alpha_{k}$ be the unique reals satisfying

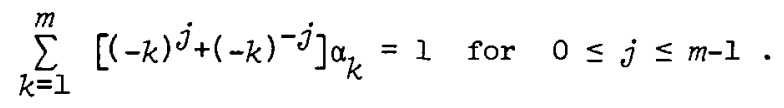

Note that the matrix of coefficients of this system is a sum of Vandermonde matrices. Its determinant, found by techniques similar to those of van der Poorten [13], is

$$
2(m !)^{1-m} \prod_{k=2}^{m} \prod_{j=1}^{k-1}(j-k)(j k-1) \neq 0 .
$$

If $\Omega=R_{+}^{n}$ or $R_{-}^{n}$, we define $P_{\Omega}: C_{0}^{m-1}(\bar{\Omega}) \rightarrow C_{0}^{m-1}\left(R^{n}\right)$ by

$$
\begin{aligned}
P_{\Omega} u(x) & =u(x) \quad \text { if } x \in \Omega, \\
& =\sum_{k=1}^{m} \alpha_{k}\left[u\left(x^{\prime},-k x_{n}\right)+u\left(x^{\prime},-k^{-1} x_{n}\right] \text { if } x \in \Omega^{\prime} .\right.
\end{aligned}
$$

As proved in Lions and Magenes [7], \$1.2.2, for a similar such reflection operator, if $s \geq 0, P_{\Omega}$ extends to a bounded operator

$$
P_{\Omega}: H^{s}(\Omega) \rightarrow H^{s}\left(\Gamma^{n}\right)
$$

Moreover, by a duality argument, the condition $s \geq 0$ can be omitted for our $P_{\Omega}$. We can now define

$$
D^{s}(\Omega)=\left(I-P_{\Omega^{\prime}} R_{\Omega^{\prime}}\right) D^{s}\left(R^{n}\right) \text { and } D^{s}(\Omega)=R_{\Omega} D^{s}\left(R^{n}\right)
$$

To show that $P_{\Omega}$ also extends to a bounded operator 
Spaces with homogeneous norms

197

$P_{\Omega}: Z^{s}(\Omega) \rightarrow Z^{s}\left(R^{n}\right)$, we obtain an equivalent norm on $Z^{s}(\Omega)$ when $s \geq 0$.

Denoting the generalized Dirichlet integral (1.7) by $[u, \Omega]_{s}^{2}$ when the integrations are over $\Omega$, we recall (Agranovich [1], pp. 19-21, for example) that

$$
\|u\|_{H(\Omega)} \sim\|u\|_{L^{2}(\Omega)}+[u, \Omega]_{s} \text { for } s \geq 0 .
$$

Now for $u \in D^{s}(\Omega)$ and $s \geq 0$, define $u_{\varepsilon}(x)=u(x / \varepsilon)$ where $\varepsilon>0$. Then $\left[u_{\varepsilon}, \Omega\right]_{s}=\varepsilon^{n / 2-s}[u, \Omega]_{s}$ and $P_{\Omega_{\varepsilon}} u_{\varepsilon}=\left(P_{\Omega}\right)_{\varepsilon}$. From $(3.5)$

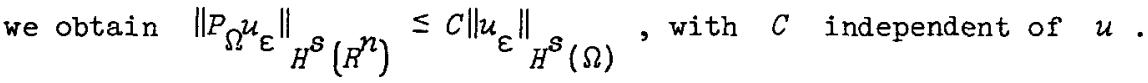
Letting $\varepsilon \rightarrow 0$ we obtain $\left[P_{\Omega} u, R^{n}\right]_{S} \leq C[u, \Omega]_{s}$. Hence

$$
\begin{aligned}
{[u, \Omega]_{s} } & \leq \inf \left\{\left[v, R^{n}\right]_{s}: v \in Z^{s}\left(R^{n}\right), R_{\Omega} v=u\right\} \\
& \leq\left[P_{\Omega} u, R^{n}\right]_{s} \\
& \leq c[u, \Omega]_{s} .
\end{aligned}
$$

As the second term in (3.8) is equivalent to $\|u\|_{Z^{s}(\Omega)}$ we have

$$
\|u\|_{z_{(\Omega)}} \sim[u, \Omega]_{s} \text { for } s \geq 0 .
$$

It also follows from (3.8) that, for $s \geq 0$,

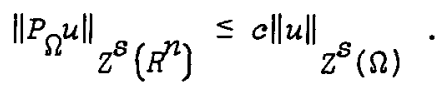

For $u \in D^{s}(\Omega)$ and $s<0$,

$$
\left\|P_{\Omega^{u}} u{ }_{H^{s}\left(R^{n}\right)} \leq c\right\| u \|_{H^{s}(\Omega)}
$$

implies

$$
\left\|\left(1+|\xi|^{2}\right)^{s / 2}\left(P_{\Omega^{u}}\right)^{\wedge}\right\|_{L^{2}\left(R^{n}\right)} \leq c\left\|\left(1+|\xi|^{2}\right)^{s / 2} \hat{v}\right\|_{L^{2}\left(R^{n}\right)}
$$

for all $v \in H^{\delta}\left(R^{n}\right)$ such that $R_{\Omega} v=u$. Scaling as above we obtain

https://doi.org/10.1017/S0004972700006018 Published online by Cambridge University Press 
$\left\||\xi|^{s}\left(P_{\Omega} u\right)^{\wedge}\right\|_{L^{2}\left(R^{n}\right)} \leq C\left\||\xi|^{s} \hat{v}\right\|_{L^{2}\left(R^{n}\right)}$ for all $v \in Z^{s}\left(R^{n}\right)\left(\subset H^{s}\left(R^{n}\right)\right)$ such that $R_{\Omega} v=u$. So (3.10) holds for $s<0$ as well.

So, for real $s, Z^{S}(\Omega)$ is complete and we have the following exact sequence

$$
0 \rightarrow q^{s}\left(\Omega^{\prime}\right) \stackrel{i}{\longrightarrow} z^{s}\left(R^{n}\right) \stackrel{R_{\Omega}}{\longrightarrow} z^{s}(\Omega) \rightarrow 0,
$$

where $i$ is the embedding and $R_{\Omega}$ has the right inverse $P_{\Omega}$. Hence $Z^{s}(\Omega)$ can be identified with $Z^{s}\left(R^{n}\right) / Z^{s}\left(\Omega^{\prime}\right)$. Moreover, as $Z^{s}(\Omega)=\left(I-P_{\Omega^{\prime}} R_{\Omega^{\prime}}\right) Z^{s}\left(R^{n}\right)$ and $Z^{s}(\Omega)=R_{\Omega} Z^{s}\left(R^{n}\right), \quad D^{s}(\Omega)$ and $D^{s}(\Omega)$ are dense subspaces of $Z^{s}(\Omega)$ and $Z^{s}(\Omega)$ respectively.

Applying the arguments of Peetre [10] for similar results in Sobolev spaces, it follows from the Paley-Wiener theorem that, for all real $s$, the following pseudo-differential operators are isomorphisms:

$$
\begin{aligned}
& \left(D_{n}-i \varepsilon_{\Omega}\left|\nabla^{\prime}\right|\right)^{s}: q^{s}(\Omega) \rightarrow L^{2}(\Omega), \\
& \left(D_{n}+i \varepsilon_{\Omega}\left|\nabla^{\prime}\right|\right)^{s}: z^{s}(\Omega) \rightarrow L^{2}(\Omega),
\end{aligned}
$$

where $\varepsilon_{\Omega}=+1$ if $\Omega=R_{+}^{n},-1$ if $\Omega=R_{-}^{n}$. The symbols of these operators, $\left(\xi_{n} \pm i\left|\xi^{\prime}\right|\right)^{s}$ are defined by taking the branch of $\zeta^{s}$ determined by $-\pi<\arg \zeta \leq \pi$. If $n=1, \xi^{\prime}=0$ and $\left(\xi_{n} \pm i 0\right)^{s}=\lim _{\delta \rightarrow 0^{+}}\left(\xi_{n} \pm i \delta\right)^{s}$. The second isomorphism in (3.12) is defined via the identification given by (3.11). As a consequence

$$
\langle u, v\rangle=\left\{\left(D_{n}-i \varepsilon_{\Omega}\left|\nabla^{\prime}\right|\right)^{s} u,\left(D_{n}+i \varepsilon_{\Omega}\left|\nabla^{\prime}\right|\right)^{-s} v\right\}_{L^{2}(\Omega)}
$$

defines a pairing on $Z^{s}(\Omega) \times Z^{-s}(\Omega)$ which is an extension of the $L^{2}$ inner product on the dense subspace $f^{S}(\Omega) \times \mathcal{D}^{-S}(\Omega)$. 


\section{The trace theorem}

As customary, let $\gamma_{j} u$, for $j=0,1, \ldots$, and $u \in c_{0}^{\infty}(\bar{\Omega})$, denote the restriction to the boundary $R^{n-1}$ of $\Omega$ of $D_{n}^{j} u=\left(\frac{1}{i} \frac{\partial}{\partial x_{n}}\right)^{j} u$.

THEOREM 4.1. For $s>\frac{1}{2}$, Let $k=\left[s+\frac{1}{2}\right]$ and $\gamma=\left(\gamma_{0}, \ldots, \gamma_{k-1}\right)$. Then $\gamma$ extends uniquely to a bounded surjection

$$
\gamma: Z^{s}(\Omega) \rightarrow \prod_{j=0}^{k-1} Z^{s-j-\frac{1}{2}}\left(R^{n-1}\right)
$$

If $s$ 丰光 $(\bmod 1)$ then $\operatorname{ker} \gamma=\mathrm{O}^{s}(\Omega)$.

Proof. That $Y$ extends uniquely to a bounded operator follows by a simple scaling argument. Next observe that on $D(\Omega)$ the norms $\|u\|$ $z^{s}\left(R^{n}\right)$ and $\|u\|_{Z(\Omega)}$ are equivalent for $s>\frac{1}{2}, s \neq \frac{2}{2}(\bmod 1)$, since the analogous result in Sobolev spaces is true (Lions, Magenes [7]) and the present result follows by scaling. Hence, for these $s, \stackrel{\circ}{Z}(\Omega)$ can be identified with a closed subspace of $Z^{s}(\Omega)$.

For

$$
g=\left(g_{0}, \ldots, g_{k-1}\right) \in \prod_{j=0}^{k-1} 0^{s-j-\frac{1}{2}}\left(R^{n-1}\right)
$$

ind $\varepsilon \geq 0$, define $E_{\varepsilon} g$ by

$\left(E_{\varepsilon} g\right)^{\wedge}(\xi)$

$$
=(2 \pi)^{\frac{1}{2}} \sum_{j=0}^{k-1}\left(\varepsilon^{2}+|\xi|^{2}\right)^{-s}\left(\varepsilon^{2}+\left|\xi^{\prime}\right|^{2}\right)^{s-(j+1) / 2} \phi_{j}\left(\xi_{n}\left(\varepsilon^{2}+\left|\xi^{\prime}\right|^{2}\right)^{-\frac{3}{2}}\right) \hat{g}_{j}\left(\xi^{\prime}\right),
$$

where $\phi_{j}(t)$ are the polynomials of degree less than or equal to $k-1$ satisfying $\int_{-\infty}^{\infty}\left(1+t^{2}\right)^{-s} t^{j^{\prime}} \phi_{j}(t) d t=\delta_{j j^{\prime}} \quad$ (see Aronszajn and Smith [3], p. 458).

As $\left|\phi_{j}(t)\right| \leq c\left(1+t^{2}\right)^{(k-1) / 2}$ it follows, as in [3], that 


$$
\left\|\left(\varepsilon^{2}+|\xi|^{2}\right)^{s / 2}\left(E_{\varepsilon} g\right)^{\wedge}\right\|_{L^{2}\left(R^{n}\right)} \leq c \sum_{j=0}^{k-1}\left\|\left(\varepsilon^{2}+\left|\xi^{\prime}\right|^{2}\right)^{\left(s-j-\frac{z}{2}\right) / 2} \hat{g}_{j}\right\|_{L^{2}\left(R^{n-1}\right)}
$$

Similarly

$$
\begin{aligned}
\left.|| \xi\right|^{s}\left(E_{\varepsilon} g\right)^{\wedge}(\xi) \mid & \leq c \sum_{j=0}^{k-1}\left(1+|\xi|^{2}\right)^{(-s+k-1) / 2}\left(1+\left|\xi^{\prime}\right|^{2}\right)^{(2 s-k-j) / 2} a_{j}\left(\xi^{\prime}\right)\left|\hat{g}_{j}\left(\xi^{\prime}\right)\right| \\
& =h(\xi)
\end{aligned}
$$

where $a_{j}\left(\xi^{\prime}\right)=\left(1+\left|\xi^{\prime}\right|^{-2}\right)^{(k-s) / 2}$ if $j=k-1$ and $s<k, a_{j}\left(\xi^{\prime}\right)=1$ otherwise, and $c$ is independent of $\varepsilon$. Also $\gamma R_{\Omega} E_{\varepsilon} g=g$.

In particular $E_{0}$ extends to a bounded operator

$$
E_{0}: \prod_{j=0}^{k-1} z^{s-j-\frac{1}{2}}\left(R^{n-1}\right) \rightarrow z^{s}\left(R^{n}\right),
$$

with $\gamma R_{\Omega} E_{0}=I$. So $\gamma$ is a surjection as required. In addition, if $s$ 光 $(\bmod 1),\left(I-R_{\Omega} E_{\varepsilon} \gamma\right) H^{s}(\Omega)=\operatorname{ker}\left(\gamma / H^{s}(\Omega)\right)=H^{s}(\Omega)$. Now $h \in L^{2}\left(R^{n}\right)$ and by the Lebesgue dominated convergence theorem, $E_{\varepsilon} g \rightarrow E_{0} g$ in $Z^{s}\left(R^{n}\right)$ as $\varepsilon \rightarrow 0$. Hence $\left(I-R_{\Omega} E_{0} \gamma\right) Z^{S}(\Omega)=\operatorname{ker} \gamma \subset \mathscr{Z}^{S}(\Omega)$. The reverse inclusion is clear. $/ /$

\section{Multiplication by smooth functions}

A property that Sobolev spaces do not in general share with spaces with homogeneous norms is that multiplication by a smooth function is continuous on the former. However

THEOREM 5.1. Let $M_{\phi}$ denote multiplication by $\phi \in C_{0}^{\infty}\left(R^{n}\right)$. For $s \geq 0, M_{\phi}$ extends to a bounded operator in $Z^{s}(\Omega)$.

Proof. Let $B$ be an open ball in $R^{n}$ containing the support of $\phi$, and let $E_{B}$ be any bounded right inverse of $R_{B}: H^{S}\left(R^{n}\right) \rightarrow H^{S}(B)$. For $u \in \mathcal{f}^{s}(\Omega)$ which is dense in $\AA^{s}(\Omega), \phi E_{B} R_{B} u=\phi u$, and 
(5.2) $\|\phi u\|_{H^{s}\left(R^{n}\right)} \leq c\left\|R_{B} u\right\|_{H^{s}(B)}$, where $c$ depends only on $\phi$.

We prove further that

$$
\left\|R_{B} u\right\|_{H^{s}(B)} \leq c\left[u, R^{n}\right]_{s}
$$

It then follows that $M_{\phi}$ extends to a bounded operator $M_{\phi}: \overbrace{}^{s}(\Omega) \rightarrow \mathscr{o}^{s}(\Omega)$ from which we obtain the theorem.

For (5.3) we note firstly that for $u \in H^{S}(B)$,

(5.4) $\quad\|u\|_{H^{s}(B)} \leq c\left([u, B]_{S}^{2}+\sum_{\mid \alpha \uparrow<s}\left|\int_{B} D^{\alpha} u d x\right|^{2}\right)^{\frac{1}{2}}$.

This generalised Poincaré inequality is proved for integer $s$ in Nečas [9], Theorem 1.1.5. The modification of the proof for other $s$ is not difficult.

Now, for $u \in \dot{u} \in H^{S}(B) / P^{S}(B)$ we can take $p \in P^{S}(B)$ such that $\int_{B} D^{\alpha}(u+p) d x=0$ for all $|\alpha|<s$. Therefore

$$
\|\dot{u}\| \leq c[u, B]_{s}
$$

Hence, for $u \in H^{s}\left(R^{n}\right)$,

$$
\left\|\left(R_{B} u\right) \cdot\right\| \leq c\left[R_{B} u, B\right]_{s} \leq c\left[u, R^{n}\right]_{s} .
$$

So (5.3) holds. For, if not, there is a sequence $\left\{\phi_{j}\right\}$ in $\delta^{s}(\Omega)$ with $\left\|R_{B} \phi_{j}\right\|_{H^{s}(B)}=1$ and $\left[\phi_{j}, R^{n}\right]_{s}<1 / j$. By (5.6) there exist $p_{j} \in P^{s}(B)$ with $R_{B} \phi_{j}+p_{j} \rightarrow 0$ in $H^{S}(B)$. Since $P^{S}(B)$ is finite dimensional, there is a convergent subsequence $\left\{p_{j_{k}}\right\}$ of $\left\{p_{j}\right\}$ with limit $p$ say. So $R_{B} \phi_{j_{k}} \rightarrow-p$ in $H^{s}(B)$, giving $\|p\|_{H^{s}(B)}=1$. But $\phi_{j} \in f^{s}(\Omega)$, so $p=0$ on $B \cap \Omega^{\prime}$ which we may assume is nonempty. Hence $p=0$, a contradiction. $\quad / /$ 


\section{Local equivalence with Sobolev spaces}

THEOREM 6.1. Let $B$ be an open ball in $R^{n}$ and $s$ real. On functions vanishing outside $B$, the norms of $Z^{s}\left(R^{n}\right)$ and $H^{s}\left(R^{n}\right)$ are equivalent. On the restrictions of those functions to $\Omega$, the norms of $Z^{s}(\Omega)$ and $H^{s}(\Omega)$ are equivalent.

Proof. The second part is a consequence of the first. The first, when $s \geq 0$, is proved in Aronszajn and Smith [3], p. 408, as noted before. If $s<0$, let $\phi \in C_{0}^{\infty}(2 B)$ with $\phi=1$ on $B$. For $u \in D^{-s}\left(R^{n}\right)$ consider $(\phi u) \cdot \epsilon H^{-s}\left(R^{n}\right) / \phi P^{-s}\left(R^{n}\right)$ and $\left(R_{2 B} u\right) \cdot \epsilon H^{-s}(2 B) / P^{-s}(2 B)$. From $(5.2)$, since $P^{-s}\left(R^{n}\right)$ is finite dimensional, and from (5.6) we obtain

$$
\|(\phi u) \cdot\| \leq c\left\|\left(R_{2 B} u\right) \cdot\right\| \leq c\left[u, R^{n}\right]_{-s} \cdot
$$

Now, for a function $v \in Z^{s}\left(R^{n}\right)$ vanishing outside $B$, $\|v\|_{Z^{s}\left(R^{n}\right)} \sim \sup _{u}\left(\left(\left|(u, v){ }_{L^{2}}\right|\right) /\left(\left[u, R^{n}\right]_{-s}\right)\right), u \in C_{0}\left(R^{n}\right)$, using (3.I), $\leq c \sup _{u}((\mid(u, v) \|) /(\|(\phi u) \cdot\|))$, using (6.2), $=c \sup _{u, p}(\mid(\phi u+\phi p, v) \|) /\left(\|\phi u+\phi p\|_{H^{-s}\left(R^{n}\right)}\right)$

$$
\begin{aligned}
& u \in C_{0}^{\infty}\left(R^{n}\right), p \in P^{-s}\left(R^{n}\right), \\
& \text { since } \bar{\phi} v=v \text { and }(\phi p, v)=(p, v)=0,
\end{aligned}
$$$$
\leq c\|v\|_{H^{s}\left(R^{n}\right)}
$$

The reverse inequality follows from the continuous embedding $Z^{s}\left(R^{n}\right) \subset H^{s}\left(R^{n}\right)$

\section{Comparison with Beppo Levi spaces}

Consider the spaces of Riesz potentials $R P^{s}\left(R^{n}\right)$ defined for $0<s<n / 2$ in the introduction. Setting $R P^{0}\left(R^{n}\right)=L^{2}\left(R^{n}\right)$ and $1 / q=1 / 2-s / n$, we have continuous embeddings 
Spaces with homogeneous norms

203

(7.1) $\quad R P^{s}\left(R^{n}\right) \subset L^{q}\left(R^{n}\right) \subset D^{\prime}\left(R^{n}\right)$, for $0 \leq s<n / 2$.

For these $s, E=R P^{s}\left(R^{n}\right)$ satisfies the required conditions for the construction of Beppo Levi spaces.

THEOREM 7.2. For $s \geq 0$ and $n \geq 2$, let $r$ be the smallest integer such that $0 \leq \sigma=s-r<n / 2$. Then $z^{s}\left(R^{n}\right)=B L_{\dot{r}}\left(R P^{\sigma}\left(R^{n}\right)\right)$. In particular, for $0 \leq s<n / 2, z^{s}\left(R^{n}\right)=R P^{s}\left(R^{n}\right)$.

Proof. Note firstly that $Z^{s}\left(R^{n}\right) \subset S^{\prime}\left(R^{n}\right) / P^{s-n / 2}\left(R^{n}\right)$ and $B L_{\dot{p}}\left(R P^{\sigma}\left(R^{n}\right)\right) \subset D^{\prime}\left(R^{n}\right) / P^{r-1}\left(R^{n}\right)$. However, $P^{s-n / 2}\left(R^{n}\right)=P^{r-1}\left(R^{n}\right)$.

Secondly, the norm on the Beppo Levi space is

$$
\left.\sum_{\mid \alpha \uparrow=r}\left[D^{\alpha} u, R^{n}\right]_{\sigma}^{2}\right]^{\frac{3}{2}}
$$

which is equivalent to the homogeneous norm. So it suffices to prove that $C_{0}^{\infty}\left(R^{n}\right) \cdot$ is dense in $B L \cdot\left(R^{n}\right)$. For this we let $\dot{u} \in B L \cdot\left(R^{n}\right)$ with $(\dot{u}, \dot{\phi})_{B L \cdot \dot{s}}=0$ for all $\left.\phi \in R_{0}^{\infty}\right)\left(R^{n}\right)$, and show $\dot{u}=0$.

We use the operator $|\nabla|^{\sigma}: S\left(R^{n}\right) \rightarrow L^{2}\left(R^{n}\right)$ and its dual $|\nabla|^{\sigma}: L^{2}\left(R^{n}\right) \rightarrow S^{\prime}\left(R^{n}\right)$, each of which is bounded. For $u \in \dot{u}$,

$$
(\dot{u}, \dot{\phi})=\sum_{\mid \alpha \uparrow=r}\left(|\nabla|^{\sigma} D^{\alpha} u,|\nabla|^{\sigma} D^{\alpha} \phi\right)_{L^{2}\left(R^{n}\right)}=\sum_{\mid \alpha \uparrow=r}\left(|\nabla|^{2 \sigma} D^{2 \alpha} u, \phi\right)=0,
$$

where $($,$) denotes the pairing between S^{\prime}\left(R^{n}\right)$ and $S\left(R^{n}\right)$. So $\sum_{|\alpha|=r}|\nabla|^{20} D^{2 \alpha} u=0$. Differentiating, we obtain for all $|\beta|=r$, $\sum_{\mid \alpha \uparrow=r}|\nabla|^{2 \sigma} D^{2 \alpha}\left(D^{\beta} u\right)=0$. As $D^{\beta} u \in L^{q}\left(R^{n}\right)$, it has a Fourier transform in $L_{\text {lac }}^{1}\left(R^{n}\right)$. Hence $\left(\sum_{|\alpha|=r}|\xi|^{20} \xi^{2 \alpha}\right)\left(D^{\beta} u\right)^{\wedge}=0$, giving $D^{\beta} u=0$. So $u \in P^{p-1}\left(R^{n}\right)$ and $\dot{u}=0$ as required.

https://doi.org/10.1017/S0004972700006018 Published online by Cambridge University Press 


\section{References}

[1] M.S. Agranovich, "Elliptic singular integro-differential operators", Russian Math. Surveys 20 (1965), no. 5, 1-121.

[2] N. Aronszajn and K.T. Smith, "Functional spaces and functional completion", Ann. Inst. Fourier (Grenoble) 6 (1955 et 1956), 125-185.

[3] N. Aronszajn and K.T. Smith, "Theory of Bessel potentials. Part I", Ann. Inst. Fourier (Grenoble) 11 (1961), 385-475.

[4] J. Deny et J.L. Lions, "Les espaces du type de Beppo Levi", Ann. Inst. Fourier (Grenoble) 5 (1953 et 1954), 305-370.

[5] Robert C. Gunning, Hugo Rossi, Analytic functions of several complex variables (Prentice-Hall, Englewood Cliffs, New Jersey, 1965).

[6] L. Hörmander et J.L. Lions, "Sur le complétion par rapport à une intégrale de Dirichlet", Math. Scand. 4 (1956), 259-270.

[7] J.L. Lions, E. Magenes, Problèmes aux limites non homogènes et applications, Tome I (Dunod, Paris, 1968). English translation: Non-homogeneous boundary value problems and applications, Volume I (translated by P. Kenneth. Die Grundlehren der mathematischen Wissenschaften, 181. Springer-Verlag, Berlin, Heidelberg, New York, 1972).

[8] B. Malgrange, "Sur l'intégrale de Dirichlet", Math. Scand. 4 (1956), 271-275.

[9] Jindrich Nečas, Les méthodes directes en théorie des équations elliptiques (Masson, Paris; Academia, Prague, 1967).

[10] Jaak Peetre, "Another approach to elliptic boundary problems", Conm. Pure App 2. Math. 14 (1961), 711-731.

[11] Jaak Peetre, "Mixed problems for higher order elliptic equations in two variables, I", Ann. Scuola Norm. Sup. Pisa (3) 15 (1961), 337-353.

[12] Jaak Peetre, "Mixed problems for higher order elliptic equations in two variables, II", Ann. Scuola Norm. Sup. Pisa (3) 17 (1963), 1-12. 
[13] A.J. van der Poorten, "Some determinants that should be better known", J. Austral. Math. Soc. Ser. A 21 (1976), 278-288.

[14] A.J. Pryde, "Elliptic partial differential equations with mixed boundary conditions" (PhD thesis, Macquarie University, New South Wales, 1976). See also: Abstract, Bull. Austral. Math. Soc. 15 (1976), 475-476.

[15] A.J. Pryde, "Second order elliptic equations with mixed boundary conditions", Bulz. Amer. Math. Soc. 83 (1977), 391-393.

[16] A.J. Pryde, "The five lemma for Banach spaces", Proc. Amer. Math. Soc. 65 (1977), 37-43.

[17] E. Shamir, "Mixed boundary value problems for elliptic equations in the plane. The $L^{p}$ theory", Ann. Scuola Norm. Sup. Pisa (3) 17 (1963), 117-139.

[18] Eliahu Shamir, "Reduced Hilbert transforms and singular integral equations", J. Analyse Math. 12 (1964), 277-305.

[19] El iahu Shamir, "Elliptic systems of singular integral operators. I. The half-space case", Trans. Amer. Math. Soc. 127 (1967), $107-124$.

[20] Elias M. Stein, Singular integrals and differentiability properties of functions (Princeton Mathematical Series, 30. Princeton University Press, Princeton, New Jersey, 1970).

[21] M.1. Vishik and G.1. Eskin, "Equations in convolutions in a bounded region", Russian Math. Surveys 20 (1965), no. 3, 85-151.

[22] M.l. Vishik and G.l. Eskin, "Elliptic equations in convolution in a bounded domain and their applications", Russion Math. Surveys 22 (1967), no. 1, 13-75.

Department of Mathematics,

University of Toronto,

Toronto,

Canada MSS $|A|$. 